\title{
Resilient degree sequences with respect to Hamilton cycles and matchings in random graphs
}

\author{
Padraig Condon Alberto Espuny Díaz Daniela Kühn* Deryk Osthus ${ }^{\dagger}$ \\ School of Mathematics \\ University of Birmingham \\ Birmingham B15 2TT, U.K. \\ \{pxc644, axe673, d.kuhn, d.osthus\}@bham.ac.uk \\ Jaehoon $\mathrm{Kim}^{\dagger}$ \\ Department of Mathematical Sciences \\ KAIST \\ Daejeon 34141, Republic of Korea \\ jaehoon.kim@kaist.ac.kr
}

Submitted: Oct 30, 2018; Accepted: Nov 24, 2019; Published: Dec 22, 2019

(C) The authors. Released under the CC BY-ND license (International 4.0).

\begin{abstract}
Pósa's theorem states that any graph $G$ whose degree sequence $d_{1} \leqslant \cdots \leqslant d_{n}$ satisfies $d_{i} \geqslant i+1$ for all $i<n / 2$ has a Hamilton cycle. This degree condition is best possible. We show that a similar result holds for suitable subgraphs $G$ of random graphs, i.e. we prove a 'resilience version' of Pósa's theorem: if $p n \geqslant C \log n$ and the $i$-th vertex degree (ordered increasingly) of $G \subseteq G_{n, p}$ is at least $(i+o(n)) p$ for all $i<n / 2$, then $G$ has a Hamilton cycle. This is essentially best possible and strengthens a resilience version of Dirac's theorem obtained by Lee and Sudakov.

Chvátal's theorem generalises Pósa's theorem and characterises all degree sequences which ensure the existence of a Hamilton cycle. We show that a natural guess for a resilience version of Chvátal's theorem fails to be true. We formulate a conjecture which would repair this guess, and show that the corresponding degree conditions ensure the existence of a perfect matching in any subgraph of $G_{n, p}$ which satisfies these conditions. This provides an asymptotic characterisation of all degree sequences which resiliently guarantee the existence of a perfect matching.
\end{abstract}

Mathematics Subject Classifications: 05C45, 05C70, 05C80.

\footnotetext{
*Supported by the EPSRC, grant no. EP/N019504/1, and by the Royal Society and the Wolfson Foundation

†Supported by the European Research Council under the European Union's Seventh Framework Programme (FP/2007-2013) / ERC Grant 306349
} 


\section{Introduction}

One of the most well-known and well-studied properties in graph theory is Hamiltonicity. We say that a graph $G$ is Hamiltonian whenever it contains a cycle which covers all of the vertices of $G$. We refer to such a cycle as a Hamilton cycle. The problem of determining whether or not a graph is Hamiltonian is NP-complete [19]. Thus, the study of Hamiltonicity focuses on finding sufficient conditions, particularly in the form of degree conditions.

In 1952, Dirac [11] proved that every $n$-vertex graph $G$ with minimum degree at least $n / 2$ is Hamiltonian. Pósa [29] strengthened this result. More specifically, a graph $G$ with degree sequence $d_{1} \leqslant \cdots \leqslant d_{n}$ such that $d_{i} \geqslant i+1$ for all $i<n / 2$ is Hamiltonian. This is best possible in the sense that the condition $d_{i} \geqslant i+1$ cannot be reduced for any $i$. Chvátal [9] generalised this further by essentially characterising all degree sequences which guarantee Hamiltonicity: a graph with degree sequence $d_{1} \leqslant \cdots \leqslant d_{n}$ is Hamiltonian if for all $i<n / 2$ we have $d_{i} \geqslant i+1$ or $d_{n-i} \geqslant n-i$.

The search for Hamilton cycles in random graphs has also been at the core of the subject (as well as the closely related problem of finding perfect matchings). Erdös and Rényi $[12,13]$ showed that the random graph $G_{n, p}$ with $p \geqslant C \log n / n$ a.a.s. contains a perfect matching (if $n$ is even and $C$ is large enough). Pósa [30] and Koršunov [22] independently showed that for the same threshold $G_{n, p}$ is a.a.s. Hamiltonian, and Komlós and Szemerédi [21] determined the exact threshold for $p$. Remarkably, one can strengthen these results to obtain the following hitting time results. Consider the following random graph process: given a vertex set of size $n$, add each of the $\left(\begin{array}{l}n \\ 2\end{array}\right)$ possible edges, one by one, chosen uniformly at random among all edges that have not been added yet. Then, Bollobás and Thomason [8] showed that a.a.s. a perfect matching appears as soon as every vertex has degree at least 1, and Ajtai, Komlós and Szemerédi [1] and Bollobás [7] independently proved that a.a.s. a Hamilton cycle appears as soon as this graph has minimum degree 2.

One more recent approach to extend the classical extremal results to random graphs is based on the following concept of resilience. The local resilience of a graph $G$ with respect to some property $\mathcal{P}$ is the maximum number $r$ such that for any subgraph $H \subseteq G$ with $\Delta(H)<r$, the graph $G \backslash H$ satisfies $\mathcal{P}$. One may view this concept as a measure of the damage an adversary can commit at each vertex of $G$, without destroying the property $\mathcal{P}$. The systematic study of local resilience was initiated by Sudakov and $\mathrm{Vu}$ [33]. Restated in this terminology, Dirac's theorem says that the local resilience of the complete graph $K_{n}$ with respect to Hamiltonicity is $\lfloor n / 2\rfloor$.

This concept of resilience naturally suggests a generalisation of Dirac's theorem in the setting of random graphs. Lee and Sudakov [25] proved that, when $p=C \log n / n$ and $C$ is sufficiently large, the local resilience of the random graph $G_{n, p}$ with respect to Hamiltonicity is a.a.s. at least $(1 / 2-\varepsilon) n p$, extending Dirac's theorem to random graphs. This improved on earlier bounds [6, 15, 33]. Very recently, Montgomery [27] as well as Nenadov, Steger and Trujić [28] independently obtained a hitting time version of this result (Nenadov, Steger and Trujić also obtained such a hitting time version for perfect 
matchings [28]).

Resilience of random graphs with respect to other properties has also been extensively studied. In particular, the containment of cycles of all possible lengths [23], $k$-th powers of cycles of all possible lengths [31], bounded degree trees [3], triangle factors [4], and bounded degree graphs $[2,17]$ have been considered. Local resilience with respect to Hamiltonicity has also been studied in other random graph models, such as binomial random directed graphs $[14,16,26]$ and random regular graphs $[5,10]$.

Lee and Sudakov [25] asked for a characterisation of the degree sequences for which the random graph $G_{n, p}$ is resilient with respect to Hamiltonicity, for $p$ close to $\log n / n$. In this paper, we partially answer this question by extending Pósa's theorem to the setting of random graphs. We also prove that the obvious extension to a Chvátal-type degree condition is false, while some modifications to those conditions suffice to force at least the containment of a perfect matching. We conjecture that such a modification is also sufficient for Hamiltonicity.

To state our results precisely, we start with the following definition, which generalises the class of graphs whose degree sequences satisfy Pósa's condition to the setting of random graphs.

Definition 1 (Pósa-resilience). Let $G=G_{n, p}$ and $\varepsilon>0$. Let $\mathcal{H}_{n, p}^{\varepsilon}$ be the collection of all $n$-vertex graphs $H$ which satisfy the following property: there is an ordering $v_{1}, \ldots, v_{n}$ of the vertices with $d_{H}\left(v_{1}\right) \geqslant \cdots \geqslant d_{H}\left(v_{n}\right)$ such that, for all $i<n / 2$,

$$
d_{H}\left(v_{i}\right) \leqslant(n-i) p-\varepsilon n p .
$$

We denote $\mathcal{H}_{n, p}^{\varepsilon}(G):=\left\{H \in \mathcal{H}_{n, p}^{\varepsilon}: H \subseteq G\right\}$. We say that $G$ is $\varepsilon$-Pósa-resilient with respect to a property $\mathcal{P}$ if $G \backslash H \in \mathcal{P}$ for all $H \in \mathcal{H}_{n, p}^{\varepsilon}(G)$.

We can now state our first main result.

Theorem 2. For every $\varepsilon>0$, there exists $C>0$ such that, for $p \geqslant C \log n / n$, a.a.s. the random graph $G_{n, p}$ is $\varepsilon$-Pósa-resilient with respect to Hamiltonicity.

Next, we consider the following definition, which generalises the class of graphs whose degree sequences satisfy Chvátal's condition to the setting of random graphs.

Definition 3 (Chvátal-resilience). Let $G=G_{n, p}$ and $\varepsilon>0$. Let $\mathcal{H}_{n, p}^{\varepsilon, 0}$ be the collection of all $n$-vertex graphs $H$ which satisfy the following property: there is an ordering $v_{1}, \cdots, v_{n}$ of the vertices with $d_{H}\left(v_{1}\right) \geqslant \cdots \geqslant d_{H}\left(v_{n}\right)$ such that, for all $i<n / 2$, either

$$
d_{H}\left(v_{i}\right) \leqslant(n-i) p-\varepsilon n p \quad \text { or } \quad d_{H}\left(v_{n-i}\right) \leqslant i p-\varepsilon n p .
$$

We denote $\mathcal{H}_{n, p}^{\varepsilon, 0}(G):=\left\{H \in \mathcal{H}_{n, p}^{\varepsilon, 0}: H \subseteq G\right\}$. We say that $G$ is $\varepsilon$-Chvátal-resilient with respect to a property $\mathcal{P}$ if $G \backslash H \in \mathcal{P}$ for all $H \in \mathcal{H}_{n, p}^{\varepsilon, 0}(G)$.

Surprisingly, unlike the case of Pósa-resilience, random graphs are not Chvátal-resilient with respect to even the containment of perfect matchings. (We actually prove a stronger result, see Theorem 16.) 
Theorem 4. For every $0<\varepsilon<10^{-6}$ there exists $C>0$ such that, for $C \log n / n \leqslant p \leqslant$ $1 / 25$, a.a.s. the random graph $G_{n, p}$ is not $\varepsilon$-Chvátal-resilient with respect to containing a perfect matching.

This leads to the following modified version of Definition 3. A related concept (i.e. a shift in the Chvátal condition) was considered by Kühn, Osthus and Treglown [24] in the setting of directed Hamilton cycles.

Definition 5 (Shifted Chvátal-resilience). Let $G=G_{n, p}$ and let $\varepsilon, \delta>0$. Let $\mathcal{H}_{n, p}^{\varepsilon, \delta}$ be the collection of all $n$-vertex graphs $H$ which satisfy the following property: there is an ordering $v_{1}, \ldots, v_{n}$ of the vertices with $d_{H}\left(v_{1}\right) \geqslant \cdots \geqslant d_{H}\left(v_{n}\right)$ such that, for all $i<n / 2$, either

$$
d_{H}\left(v_{i}\right) \leqslant(n-i) p-\varepsilon n p
$$

or

$$
d_{H}\left(v_{n-i-\delta n}\right) \leqslant i p-\varepsilon n p .
$$

We denote $\mathcal{H}_{n, p}^{\varepsilon, \delta}(G):=\left\{H \in \mathcal{H}_{n, p}^{\varepsilon, \delta}: H \subseteq G\right\}$. We say that $G$ is $(\varepsilon, \delta)$-Chvátal-resilient with respect to a property $\mathcal{P}$ if $G \backslash H \in \mathcal{P}$ for all $H \in \mathcal{H}_{n, p}^{\varepsilon, \delta}(G)$.

Note that (3) is never satisfied for $i<\varepsilon n$. The conditions (2) and (3) together imply that

$$
d_{H}(v) \leqslant(1-\varepsilon) n p
$$

for all $H \in \mathcal{H}_{n, p}^{\varepsilon, \delta}$ and all vertices $v$ of $H$. As $\mathcal{H}_{n, p}^{\varepsilon} \subseteq \mathcal{H}_{n, p}^{\varepsilon, \delta}$, the same bound holds when considering $\varepsilon$-Pósa-resilience.

With this new definition of shifted Chvátal-resilience we can obtain the following version of Chvátal's theorem for random graphs with respect to the containment of perfect matchings.

Theorem 6. For every $\varepsilon>0$, there exists $C>0$ such that, for $p \geqslant C \log n / n$, a.a.s. the random graph $G_{n, p}$ is $(\varepsilon, \varepsilon)$-Chvátal-resilient with respect to containing a perfect matching if $n$ is even.

We conjecture that Theorem 6 also holds if perfect matchings are replaced by Hamilton cycles.

Conjecture 7. For every $\varepsilon>0$, there exists $C>0$ such that, for $p \geqslant C \log n / n$, a.a.s. the random graph $G_{n, p}$ is $(\varepsilon, \varepsilon)$-Chvátal-resilient with respect to Hamiltonicity.

The following simple construction shows that this statement, if true, is essentially best possible. Let $G=G_{n, p}$ with $p \geqslant C \log n / n$ for some sufficiently large $C$. Given any $\varepsilon n \leqslant i<n / 2$, fix disjoint sets $X, Y \subseteq V$ of sizes $i$ and $n-i$, respectively, and let $H$ be the induced bipartite subgraph between $X$ and $Y$. One can then prove that a.a.s.

$$
d_{H}(x) \leqslant(n-i) p+\varepsilon n p \quad \text { and } \quad d_{H}(y) \leqslant i p+\varepsilon n p
$$


for all $x \in X$ and $y \in Y$. Thus, $H$ is 'close' to satisfying the conditions of Definition 5, and it is clear that $G \backslash H$ is not Hamiltonian since it is disconnected. The same construction shows that Theorem 2 is essentially best possible (in the sense that we cannot significantly relax the degree condition) and that Theorem 6 is essentially best possible when considering odd $i$.

Investigating resilience with respect to degree sequences is natural not only for perfect matchings and Hamilton cycles, but also for other properties. Several results on degree sequences forcing given substructures have been obtained in the classical setting (see e.g. [32, 34] for such results involving Pósa-type degree sequences and [20] for Chvátaltype degree sequences). It would be interesting to see if one can obtain resilience versions (for random graphs) of some of these results.

\section{Preliminaries}

\section{$2.1 \quad$ Notation}

For $n \in \mathbb{N}$, we denote $[n]:=\{1, \ldots, n\}$. The constants which appear in hierarchies are chosen from right to left. That is, whenever we use a hierarchy $0<1 / n \ll a \ll b \leqslant 1$, we mean that there exist non-decreasing functions $f:[0,1) \rightarrow[0,1)$ and $g:[0,1) \rightarrow[0,1)$ such that the result holds for all $0 \leqslant a, b \leqslant 1$ and all $n \in \mathbb{N}$ with $a \leqslant f(b)$ and $1 / n \leqslant g(a)$. We will not calculate these functions explicitly.

We use a.a.s. as an abbreviation for asymptotically almost surely. Whenever we claim that a result holds a.a.s. for $G_{n, p}$, we mean that the probability that our result holds tends to one as $n$ tends to infinity. For the purpose of clarity, we will ignore rounding issues when dealing with asymptotic statements, whenever the values we consider tend to infinity with $n$.

Given an $n$-vertex graph $G$ we define $e(G):=|E(G)|$. Given a set $A \subseteq V(G)$ we denote by $e_{G}(A)$ the number of edges in $G$ whose endpoints are both in $A$. Given another set $B \subseteq V(G)$ we denote by $E_{G}(A, B)$ the set of edges of $G$ with one endpoint in $A$ and the other in $B$ (note that $A$ and $B$ are allowed to have a nonempty intersection), and $e_{G}(A, B):=\left|E_{G}(A, B)\right|$. Given any $v \in V(G)$, we will write $e_{G}(v, A):=e_{G}(\{v\}, A)$. Sometimes it will be useful to consider $e_{G}^{\prime}(A, B):=e_{G}(A, B)+e_{G}(A \cap B)$. We will often refer to the graph $G[V(G) \backslash A]$, which we denote as $G-A$. If $A$ and $B$ are disjoint, the notation $G[A, B]$ will refer to the induced bipartite subgraph with vertex classes $A$ and $B$. We denote the neighbourhood of $A$ as $N_{G}(A):=\left\{v \in V(G): e_{G}(\{v\}, A)>0\right\}$. Given a vertex $v \in V(G)$ we define its degree as $d_{G}(v):=\left|N_{G}(\{v\})\right|$. We denote the minimum degree in a set of vertices as $\delta_{G}(A):=\min \left\{d_{G}(v): v \in A\right\}$, and the maximum degree as $\Delta(G):=\max \left\{d_{G}(v): v \in V(G)\right\}$. We often consider the sequence of degrees of the vertices of $G$ ordered increasingly, and refer to it as the degree sequence of $G$.

The binomial random graph $G_{n, p}$ is obtained by adding each of the edges of a complete graph on $n$ vertices with probability $p$, independently of the other edges. We will always denote the vertex set of $G_{n, p}$ by $V$. We use $G_{n, m, p}$ for a random bipartite graph with vertex classes of size $n$ and $m$, respectively; each edge between the classes is added with 
probability $p$ independently of every other edge, as above. Whenever we consider a random bipartite graph between vertex sets $A$ and $B$, we also refer to this model as $G_{A, B, p}$.

\subsection{Tools for random graphs}

We will need the following Chernoff bound (see e.g. [18, Corollary 2.3]).

Lemma 8. Let $X$ be the sum of $n$ independent Bernoulli random variables and let $\mu:=$ $\mathbb{E}[X]$. Then, for all $0 \leqslant \delta \leqslant 1$ we have that $\mathbb{P}[X \neq(1 \pm \delta) \mu] \leqslant 2 e^{-\delta^{2} \mu / 3}$.

The following lemmas are standard results for random graphs. They can be proved using Chernoff bounds and the fact that the considered random variables follow binomial distributions.

Lemma 9. There exist constants $C, c>0$ such that for any $p \geqslant C \log n / n$ the random graph $G=G_{n, p}$ a.a.s. satisfies that for all $X, Y \subseteq V$ we have

$$
\left.\left|e_{G}(X, Y)-\right| X|| Y|p+| X \cap Y\right|^{2} p / 2 \mid \leqslant c \sqrt{|X||Y| n p}
$$

and

$$
\left|e_{G}^{\prime}(X, Y)-\right| X|| Y|p| \leqslant c \sqrt{|X||Y| n p} .
$$

Lemma 10. For every $\eta>0$, there exists a constant $C$ such that for $p \geqslant C \log n / n$ the random graph $G=G_{n, p}$ a.a.s. satisfies that $d_{G}(v)=(1 \pm \eta)$ np for all $v \in V$.

Lemma 11. Let $A$ and $B$ be two disjoint sets of vertices with $|A|=n,|B|=m$ and $m=\Theta(n)$. For every $\eta>0$, there exists a constant $C$ such that, for $p \geqslant C \log n / n$, the random graph $G=G_{A, B, p}$ a.a.s. satisfies that for each $v \in A$ we have $d_{G}(v)=(1 \pm \eta) m p$.

We now prove some properties of the subgraphs of the random graphs which satisfy the conditions of Definition 5.

Proposition 12. For every $0<\varepsilon<1$, there exists $C>0$ such that for $p \geqslant C \log n / n$ the random graph $G=G_{n, p}$ a.a.s. satisfies that, for all $H \in \mathcal{H}_{n, p}^{\varepsilon, \varepsilon}(G)$ and $G^{\prime}:=G \backslash H$, the following hold:

(i) For each $X \subseteq V$, we have $\left|N_{G^{\prime}}(X)\right| \geqslant \min \left\{\varepsilon|X| n p / 2\right.$, $\left.\varepsilon n(\log n)^{-1 / 4} / 2\right\}$.

(ii) For each $X \subseteq V$ with $|X| \geqslant n(\log n)^{-1 / 2}$, we have that

$$
\left|N_{G^{\prime}}(X)\right|>\left(1-\varepsilon^{2} / 10\right) p^{-1} \delta_{G^{\prime}}(X) .
$$

In particular, $\left|N_{G^{\prime}}(X)\right| \geqslant \varepsilon n / 2$.

(iii) $G^{\prime}$ is connected. 
Proof. Choose a number $0<\eta \ll \varepsilon$. Consider the event that for all $v \in V$ we have

$$
d_{G}(v)=(1 \pm \eta) n p
$$

and for all $X, Y \subseteq V$ with $|X| \geqslant n(\log n)^{-1 / 2}$ and $|Y| \geqslant \eta n$ we have

$$
e_{G}^{\prime}(X, Y)=(1 \pm \eta)|X||Y| p .
$$

Throughout the proof, we condition on the event that (5) and (6) hold. Note that Lemmas 9 and 10 imply that such an event a.a.s. occurs.

(i). To prove (i), a simple calculation (see e.g. [25, Proposition 2.5(i)]) shows that a.a.s. for all $X \subseteq V$ of size at most $\left\lceil(\log n)^{-1 / 4} p^{-1}\right\rceil$,

$$
\left|N_{G}(X)\right| \geqslant(1-\varepsilon / 2)|X| n p .
$$

As $H \in \mathcal{H}_{n, p}^{\varepsilon, \varepsilon}$, (4) together with (7) implies

$$
\left|N_{G^{\prime}}(X)\right| \geqslant\left|N_{G}(X)\right|-(1-\varepsilon) n p|X| \geqslant \varepsilon n p|X| / 2 .
$$

Given a set $X \subseteq V$ of size at least $(\log n)^{-1 / 4} p^{-1}$, we can choose a subset $X^{\prime} \subseteq X$ of size $\left\lceil(\log n)^{-1 / 4} p^{-1}\right\rceil$, and apply the bound above to obtain $\left|N_{G^{\prime}}(X)\right| \geqslant\left|N_{G^{\prime}}\left(X^{\prime}\right)\right| \geqslant$ $\varepsilon n(\log n)^{-1 / 4} / 2$. This proves (i).

(ii). As $H \in \mathcal{H}_{n, p}^{\varepsilon, \varepsilon},(5)$ together with (4) implies that $\delta_{G^{\prime}}(X) \geqslant(\varepsilon-\eta) n p$. For each $X \subseteq V$, we have

$$
e_{G^{\prime}}^{\prime}(X, V) \geqslant|X| \delta_{G^{\prime}}(X) .
$$

Suppose that there is a set $X \subseteq V$ with $|X| \geqslant n(\log n)^{-1 / 2}$ and $\left|N_{G^{\prime}}(X)\right| \leqslant(1-$ $\left.\varepsilon^{2} / 10\right) p^{-1} \delta_{G^{\prime}}(X)$. Let $Y \subseteq V$ be a set containing $N_{G^{\prime}}(X)$ and such that $|Y|=(1-$ $\left.\varepsilon^{2} / 10\right) p^{-1} \delta_{G^{\prime}}(X) \geqslant \eta n$. Hence, (6) implies that

$$
e_{G}^{\prime}(X, Y) \leqslant(1+\eta) p|X|\left(1-\varepsilon^{2} / 10\right) p^{-1} \delta_{G^{\prime}}(X) \leqslant\left(1-\varepsilon^{2} / 20\right)|X| \delta_{G^{\prime}}(X) \stackrel{(8)}{<} e_{G^{\prime}}^{\prime}(X, V),
$$

a contradiction to the fact that $N_{G^{\prime}}(X) \subseteq Y$. In particular, as $\delta_{G^{\prime}}(X) \geqslant(\varepsilon-\eta) n p$, we have $\left|N_{G^{\prime}}(X)\right| \geqslant\left(1-\varepsilon^{2} / 10\right)(\varepsilon-\eta) n \geqslant \varepsilon n / 2$. This proves (ii).

(iii). Condition on the event that statements (i) and (ii) hold, in addition to (5) and (6). Assume that $G^{\prime}$ is not connected, and let $X \subseteq V$ be a (connected) component of $G^{\prime}$ such that $|X| \leqslant n / 2$. Note that $\left|N_{G^{\prime}}(X)\right|=|X|$. As (i) and (ii) both hold, it is easy to see that $|X| \geqslant \varepsilon n / 2$. Let $m:=|X|-\varepsilon n / 4 \geqslant \varepsilon n / 4$.

As $H \in \mathcal{H}_{n, p}^{\varepsilon, \varepsilon}$, by Definition 5 there exists a labelling $v_{1}, \ldots, v_{n}$ of $V$ with $d_{H}\left(v_{1}\right) \geqslant$ $\cdots \geqslant d_{H}\left(v_{n}\right)$ such that we have either

$$
d_{H}\left(v_{m}\right) \leqslant(n-m) p-\varepsilon n p \quad \text { or } \quad d_{H}\left(v_{n-m-\varepsilon n}\right) \leqslant m p-\varepsilon n p .
$$

If the former is true, then there exists a set $X^{\prime} \subseteq X \cap\left\{v_{m}, \ldots, v_{n}\right\}$ with $\left|X^{\prime}\right|=\varepsilon n / 4$ and

$$
\delta_{G^{\prime}}\left(X^{\prime}\right) \stackrel{(5)}{\geqslant}(1-\eta) n p-(n-m) p+\varepsilon n p \geqslant m p+\varepsilon n p / 2 .
$$


Then, (ii) ensures that $\left|N_{G^{\prime}}\left(X^{\prime}\right)\right| \geqslant\left(1-\varepsilon^{2} / 10\right)(m+\varepsilon n / 2) \geqslant m+\varepsilon n / 3>|X|$, a contradiction to the fact that $X$ is a component of $G^{\prime}$.

Hence, we may assume that the latter of (9) holds. In this case, there are at least $m+\varepsilon n \geqslant|X|+\varepsilon n / 2$ vertices $v$ with $d_{H}(v) \leqslant m p-\varepsilon n p$, hence there exists a set $Y \subseteq$ $\left\{v_{n-m-\varepsilon n}, \ldots, v_{n}\right\} \backslash X$ with $|Y| \geqslant \varepsilon n / 2$ and

$$
\delta_{G^{\prime}}(Y) \stackrel{(5)}{\geqslant}(1-\eta) n p-m p+\varepsilon n p \geqslant(n-m) p+\varepsilon n p / 2 .
$$

Then, (ii) ensures that $\left|N_{G^{\prime}}(V \backslash X)\right| \geqslant\left|N_{G^{\prime}}(Y)\right| \geqslant\left(1-\varepsilon^{2} / 10\right)(n-m+\varepsilon n / 2) \geqslant n-m+$ $\varepsilon n / 3>|V \backslash X|$, a contradiction to the fact that $X$ is a component of $G^{\prime}$.

\section{Chvátal-type resilience for matchings in random graphs}

Proof of Theorem 6 . Let $0<1 / n \ll 1 / C \ll \eta \ll \varepsilon \ll 1$ and $1 / c<1$, where $n$ is even and $c$ is the constant given by Lemma 9 . We condition on the event that $G=G_{n, p}$ satisfies the assertions of Lemma 9, Lemma 10 and Proposition 12 with the chosen constants $\varepsilon$, $\eta, C$ and $c$, which happens a.a.s. We will show that all such $G$ are $(\varepsilon, \varepsilon)$-Chvátal-resilient with respect to containing a perfect matching. Let $H \in \mathcal{H}_{n, p}^{\varepsilon, \varepsilon}(G)$ and let $G^{\prime}:=G \backslash H$. Let $v_{1}, \ldots, v_{n}$ be an ordering of the vertices as in Definition 5 . Let $D(H):=\left\{v_{\lceil n / 2\rceil}, \ldots, v_{n}\right\}$. In particular, by Lemma 10 we have that

$$
\delta_{G^{\prime}}(D(H)) \geqslant(1+\varepsilon) n p / 2 .
$$

By Tutte's theorem, it suffices to show that, for any vertex set $U \subseteq V$, the number of odd components of $G^{\prime}-U$ is at most $|U|$ (here a component is odd if it contains an odd number of vertices). As we conditioned on the assertion of Proposition 12(iii) and since $n$ is even, this holds if $U$ is the empty set.

Hence, we will prove that, for any non-empty $U \subseteq V$, the number of (not necessarily odd) components of $G^{\prime}-U$ is at most $|U|$. As each component of $G^{\prime}-U$ has at least one vertex, we may further assume that $|U|<n / 2$.

Let $U \subseteq V$ with $|U|<n / 2$ and let $k$ be the total number of components of $G^{\prime}-U$. To derive a contradiction, assume that $k>|U|$; in particular, $k \geqslant 2$. Enumerate the components in $G^{\prime}-U$ as $C_{1}, \ldots, C_{k}$ with $\left|C_{1}\right| \leqslant\left|C_{2}\right| \leqslant \cdots \leqslant\left|C_{k}\right|$. For each $S \subseteq[k]$, let $C_{S}:=\bigcup_{i \in S} C_{i}$. We consider the cases where $|U|$ is small and large separately.

Case 1: $|U| \leqslant \varepsilon n / 10$.

First, we prove that $\left|C_{k}\right|$ is large in this case.

Claim 13. We have $\left|C_{k}\right|>n / 2$.

Proof. Suppose otherwise that $\left|C_{k}\right| \leqslant n / 2$. Let

$$
\mathcal{S}:=\left\{S \subseteq[k]:\left|C_{S} \cap D(H)\right| \geqslant \varepsilon n\right\} .
$$

Let $S^{*} \in \mathcal{S}$ be a set in $\mathcal{S}$ with the minimum $\left|C_{S^{*}}\right|$. We claim that $\left|C_{S^{*}}\right| \leqslant n / 2$. Indeed, suppose this is not the case. Then, we have $\left|S^{*}\right| \geqslant 2$. As a partition of $S^{*}$ into two nonempty sets yields two disjoint sets not in $\mathcal{S}$, we have $\left|C_{S^{*}} \cap D(H)\right|<2 \varepsilon n$. Thus $C_{[k] \backslash S^{*}}$ 
satisfies that $\left|C_{[k] \backslash S^{*}}\right| \leqslant n / 2$ and $\left|C_{[k] \backslash S^{*}} \cap D(H)\right| \geqslant n / 2-3 \varepsilon n$ so we have $[k] \backslash S^{*} \in \mathcal{S}$, which contradicts the minimality of $C_{S^{*}}$. Hence we have $\left|C_{S^{*}}\right| \leqslant n / 2$.

Let $D:=C_{S^{*}} \cap D(H)$. As we have $|D| \geqslant \varepsilon n$, by (10) and Proposition 12(ii) we have

$$
\left|N_{G^{\prime}}(D)\right|>\left(1-\varepsilon^{2} / 10\right)(1+\varepsilon) n / 2>n / 2+|U| .
$$

It follows that at least one vertex $v \in D \subseteq C_{S^{*}}$ is adjacent to a vertex $u \in C_{[k] \backslash S^{*}}$, a contradiction. This proves the claim.

Let $\ell:=\left|C_{[k-1]}\right|$. Note that $\ell<n / 2$.

Claim 14. We have $\ell<\varepsilon n / 6$.

Proof. Assume otherwise that $\ell \geqslant \varepsilon n / 6$.

First, assume that $H$ satisfies (2) for all $i \in[\ell] \backslash[\ell-\varepsilon n / 8]$. Note that the set $C^{\prime}:=C_{[k-1]} \backslash\left\{v_{1}, \ldots, v_{\ell-\varepsilon n / 8}\right\}$ satisfies $\left|C^{\prime}\right| \geqslant \varepsilon n / 8$. Because $G$ satisfies the assertion of Lemma 10 and $v_{\ell-\varepsilon n / 8+1}$ satisfies (2) for $H$, we have

$\delta_{G^{\prime}}\left(C^{\prime}\right) \geqslant \delta_{G}(V)-d_{H}\left(v_{\ell-\varepsilon n / 8+1}\right) \geqslant(1-\eta) n p-((n-\ell+\varepsilon n / 8-1) p-\varepsilon n p) \geqslant \ell p+3 \varepsilon n p / 4$.

As $G^{\prime}$ satisfies the assertion of Proposition 12(ii), we have

$$
\left|N_{G^{\prime}}\left(C_{[k-1]}\right)\right| \geqslant\left|N_{G^{\prime}}\left(C^{\prime}\right)\right| \geqslant\left(1-\varepsilon^{2} / 10\right)(\ell+3 \varepsilon n / 4) \geqslant \ell+\varepsilon n / 2>\left|C_{[k-1]}\right|+|U|,
$$

a contradiction as $C_{k}$ and $C_{[k-1]}$ are disconnected in $G^{\prime}-U$.

So suppose that there is an index $j \in[\ell] \backslash[\ell-\varepsilon n / 8]$ such that $H$ does not satisfy (2) for $j$. We have that the set $C^{\prime \prime}:=C_{k} \backslash\left\{v_{1}, \ldots, v_{n-j-\varepsilon n}\right\}$ satisfies

$$
\left|C^{\prime \prime}\right| \geqslant\left|C_{k}\right|-(n-j-\varepsilon n)=n-\ell-|U|-(n-j-\varepsilon n) \geqslant \varepsilon n / 4 .
$$

Here, we obtain the final inequality as $|U| \leqslant \varepsilon n / 10$ and $j \geqslant \ell-\varepsilon n / 8$. Moreover, because $G$ satisfies the assertion of Lemma 10, the fact that (3) holds for $j$ implies that

$$
\delta_{G^{\prime}}\left(C^{\prime \prime}\right) \geqslant \delta_{G}(V)-d_{H}\left(v_{n-j-\varepsilon n+1}\right) \geqslant(1-\eta) n p-(j-\varepsilon n) p \geqslant(n-j+\varepsilon n / 2) p .
$$

As $G^{\prime}$ satisfies the assertion of Proposition 12(ii), this shows that

$$
\left|N_{G^{\prime}}\left(C^{\prime \prime}\right)\right|>\left(1-\varepsilon^{2} / 10\right)(n-j+\varepsilon n / 2) \geqslant n-\ell+\varepsilon n / 6>\left|C_{k}\right|+|U|,
$$

a contradiction to the fact that $C_{k}$ is a component of $G^{\prime}-U$. This proves the claim.

It follows from the previous two claims that $G^{\prime}-U$ has one 'giant' component $C_{k}$, containing more than $(1-\varepsilon / 3) n$ vertices. The following claim will give us the desired contradiction.

Claim 15. For any set $W \subseteq V$ with $|W|<\varepsilon n / 6$, we have that $\left|N_{G^{\prime}}(W)\right|>2|W|$. 
Proof. If $|W| \leqslant n(\log n)^{-1 / 2}$, then, as $G^{\prime}$ satisfies the assertion of Proposition 12(i), we have

$$
\left|N_{G^{\prime}}(W)\right| \geqslant \min \left\{\frac{1}{2} \varepsilon|W| n p, \frac{1}{2} \varepsilon n(\log n)^{-1 / 4}\right\}>2|W| .
$$

If we have $n(\log n)^{-1 / 2} \leqslant|W|<\varepsilon n / 6$, then, because $G^{\prime}$ satisfies the assertion of Proposition 12(ii), we have

$$
\left|N_{G^{\prime}}(W)\right| \geqslant \varepsilon n / 2>2|W|
$$

Recall that $|U| \leqslant k-1 \leqslant \ell$. As $C_{k}$ and $C_{[k-1]}$ are disconnected in $G^{\prime}-U$, we have $\left|N_{G^{\prime}}\left(C_{[k-1]}\right)\right| \leqslant\left|C_{[k-1]}\right|+|U| \leqslant 2 \ell$. However, by Claims 14 and 15 , we have $\left|N_{G^{\prime}}\left(C_{[k-1]}\right)\right|>$ $2 \ell$, a contradiction. This concludes Case 1.

Case 2: $|U|>\varepsilon n / 10$.

Let $S:=\left\{i \in[k]:\left|C_{i}\right|<2 \sqrt{n}\right\}$ and $t:=|U|$. We first claim that

$$
t-\sqrt{n} \leqslant\left|C_{S}\right|
$$

Indeed, suppose otherwise. As $k>t$ and each component of $G^{\prime}-U$ contains at least one vertex, we have

$$
|[k] \backslash S|>t-|S| \geqslant t-\left|C_{S}\right|>\sqrt{n} .
$$

Hence, $\left|C_{[k] \backslash S}\right| \geqslant 2 \sqrt{n} \cdot|[k] \backslash S|>2 n$, a contradiction. Thus $t-\sqrt{n} \leqslant\left|C_{S}\right|$.

As $G$ satisfies the assertion of Lemma 9, by the definition of $S$ we have

$$
\begin{aligned}
e_{G^{\prime}}\left(C_{S}\right) & \leqslant \sum_{i \in S} e_{G}\left(C_{i}\right) \leqslant \sum_{i \in S}\left(\left|C_{i}\right|^{2} p+c\left|C_{i}\right| \sqrt{n p}\right) \leqslant\left(\sum_{i \in S}\left|C_{i}\right|\right)(2 \sqrt{n} p+c \sqrt{n p}) \\
& \leqslant\left|C_{S}\right| \cdot 4 c \sqrt{n p} \leqslant 4 c n^{3 / 2} p^{1 / 2} \leqslant \eta n^{2} p .
\end{aligned}
$$

We also claim that

$$
C_{S} \text { does not contain any set } C^{\prime} \text { with }\left|C^{\prime}\right| \geqslant \varepsilon n / 20 \text { and } \delta_{G^{\prime}}\left(C^{\prime}\right) \geqslant t p+\varepsilon n p / 2 \text {. }
$$

Indeed, suppose $C_{S}$ contains such a set $C^{\prime}$. By (12) we have that

$$
e_{G^{\prime}}\left(C^{\prime}, U\right) \geqslant\left|C^{\prime}\right| \delta_{G^{\prime}}\left(C^{\prime}\right)-2 e_{G^{\prime}}\left(C_{S}\right)>\left|C^{\prime}\right| t p+\varepsilon^{2} n^{2} p / 50 .
$$

On the other hand, as $G$ satisfies the assertion of Lemma 9, we have

$$
e_{G^{\prime}}\left(C^{\prime}, U\right) \leqslant e_{G}\left(C^{\prime}, U\right) \leqslant\left|C^{\prime}\right| t p+c \sqrt{\left|C^{\prime}\right| \operatorname{tnp}} \leqslant\left|C^{\prime}\right| t p+\varepsilon^{2} n^{2} p / 100,
$$

a contradiction. Hence, such a set $C^{\prime}$ does not exist.

Suppose that $H$ satisfies (2) for all $i \in[t] \backslash[t-\varepsilon n / 10]$. As $G$ satisfies the assertion of Lemma 10 and by (11), the set $C^{\prime \prime}:=C_{S} \backslash\left\{v_{1}, \ldots, v_{t-\varepsilon n / 10}\right\}$ satisfies $\left|C^{\prime \prime}\right| \geqslant\left|C_{S}\right|-t+$ $\varepsilon n / 10 \geqslant \varepsilon n / 20$ and

$$
\delta_{G^{\prime}}\left(C^{\prime \prime}\right) \geqslant(1-\eta) n p-(n-t-9 \varepsilon n / 10) p \geqslant t p+\varepsilon n p / 2,
$$


a contradiction to (13).

Hence, there exists $j \in[t] \backslash[t-\varepsilon n / 10]$ such that $H$ does not satisfy (2) for $j$. By (3) and Lemma 10, this means that

$$
\delta_{G}(V)-d_{H}\left(v_{n-t-9 \varepsilon n / 10-1}\right) \geqslant(1-\eta) n p-(j-\varepsilon n) p \geqslant(n-t+4 \varepsilon n / 5) p .
$$

Therefore, the set $R:=V \backslash\left(U \cup\left\{v_{1}, \ldots, v_{n-t-9 \varepsilon n / 10}\right\}\right)$ satisfies $|R| \geqslant 9 \varepsilon n / 10$ and $\delta_{G^{\prime}}(R)>$ $(n-t+4 \varepsilon n / 5) p$. As $t \leqslant n / 2$, we have $\delta_{G^{\prime}}(R) \geqslant t p+\varepsilon n p / 2$. Hence, we conclude $\left|R \cap C_{S}\right|<$ $\varepsilon n / 20$, otherwise we have a contradiction to (13).

Hence, $R^{\prime}:=R \cap C_{[k] \backslash S}$ satisfies $\left|R^{\prime}\right| \geqslant 4 \varepsilon n / 5$. As $G^{\prime}$ satisfies the assertion of Proposition 12(ii), we conclude that

$$
\left|N_{G^{\prime}}\left(R^{\prime}\right)\right| \geqslant\left(1-\varepsilon^{2} / 10\right)(n-t+4 \varepsilon n / 5) \geqslant n-t+\varepsilon n / 2 \stackrel{(11)}{>}\left|V \backslash C_{S}\right| .
$$

This is a contradiction as $R^{\prime}$ lies inside $C_{[k] \backslash S}$, which is disconnected from $C_{S}$ in $G^{\prime}-U$.

We now show that Theorem 6 is best possible in the sense that $(\varepsilon, \varepsilon)$-Chvátal-resilience cannot be improved to allow for $\left(\varepsilon,(3 n p)^{-1}\right)$-Chvátal-resilience. That is, unlike the classical theorem of Chvátal, the random graphs analogue requires an extra shift in the indices whenever we veer from a Pósa degree sequence.

Given an $n$-vertex graph $G$, we say that $G$ contains an optimal matching if it has a matching of size $\lfloor n / 2\rfloor$. In particular, if $G$ does not contain an optimal matching, then $G$ cannot be Hamiltonian. Note that Theorem 16 implies Theorem 4.

Theorem 16. For every $0<\varepsilon<10^{-6}$ there exists $C>0$ such that, for any $C \log n / n \leqslant$ $p \leqslant 1 / 25$, the random graph $G=G_{n, p}$ is a.a.s. not $\left(\varepsilon,\left\lceil(3 p)^{-1}\right\rceil / n\right)$-Chvátal-resilient with respect to containing an optimal matching.

The proof strategy is as follows. We consider $G_{n, p}$ and remove appropriate edges to create a graph $G^{\prime}$ having an independent set $X$ with $\left|N_{G^{\prime}}(X)\right|<|X|-1$. This ensures that $G^{\prime}$ does not contain an optimal matching. We conclude the proof by showing that $G \backslash G^{\prime} \in \mathcal{H}_{n, p}^{\left.\varepsilon, \Gamma(3 p)^{-1}\right\rceil / n}$.

Proof. Let $1 / n \ll 1 / C \ll \eta \ll \varepsilon<10^{-6}$. Let $Y \subseteq V$ be any set of vertices of size $\left\lfloor((1+\eta) 2 p)^{-1}\right\rfloor$. Now expose all edges of $G$ incident to $Y$. Let $\mathcal{E}_{1}$ be the event that, for each vertex $y \in Y$, we have

$$
d_{G}(y)=(1 \pm \eta) n p .
$$

Note that Lemma 10 implies that $\mathcal{E}_{1}$ happens a.a.s. We condition on the event $\mathcal{E}_{1}$. Thus we have

$$
\left|N_{G}(Y)\right| \leqslant \sum_{y \in Y} d_{G}(y) \leqslant|Y|(1+\eta) n p \leqslant n / 2 .
$$

Fix disjoint sets $X, U \subseteq V \backslash\left(Y \cup N_{G}(Y)\right)$ with $|X|=100$ en and $|U|=|X|-2$. Now, expose all remaining edges of $G$ (i.e. those not incident to $Y$ ). Let $\mathcal{E}_{2}$ be the event that the following hold for all $v \in V \backslash Y$ and $Z \in\{X, U\}$ :

$$
\begin{aligned}
e_{G}(v, Z) & =(1 \pm \eta)|Z| p, \\
d_{G}(v) & =(1 \pm \eta) n p .
\end{aligned}
$$


By Lemmas 10 and 11, the event $\mathcal{E}_{2}$ happens a.a.s. under conditioning on $\mathcal{E}_{1}$. We condition on the event that both $\mathcal{E}_{1}$ and $\mathcal{E}_{2}$ hold, i.e. that $G$ satisfies (14)-(16). We will show that every such $G$ is not $\left(\varepsilon,\left\lceil(3 p)^{-1}\right\rceil / n\right)$-Chvátal-resilient with respect to containing an optimal matching.

We construct a spanning subgraph $G^{\prime}$ of $G$ by deleting all edges in $G[X]$ and all edges in $G[X, V \backslash(X \cup U)]$. From the construction, $X$ is an independent set of $G^{\prime}$ and $N_{G^{\prime}}(X) \subseteq U$. Thus, $\left|N_{G^{\prime}}(X)\right| \leqslant|U|<|X|-1$, hence $G^{\prime}$ does not contain an optimal matching.

Let $\gamma:=\left\lceil(3 p)^{-1}\right\rceil / n$. Now it suffices to show that $H:=G \backslash G^{\prime} \in \mathcal{H}_{n, p}^{\varepsilon, \gamma}$. From the construction, it is easy to see that (15) and (16) imply that, for all $u \in Y \cup U, x \in X$ and $v \in V \backslash(X \cup U \cup Y)$,

$$
d_{H}(u)=0, \quad d_{H}(x)=(1-100 \varepsilon \pm 3 \eta) n p \quad \text { and } \quad d_{H}(v)=(1 \pm \eta) 100 \varepsilon n p .
$$

Let $v_{1}, \ldots, v_{n}$ be an ordering of $V$ with $d_{H}\left(v_{1}\right) \geqslant \cdots \geqslant d_{H}\left(v_{n}\right)$. Observe that, in this ordering, $X=\left\{v_{1}, \ldots, v_{|X|}\right\}$ and $U \cup Y=\left\{v_{n-|U \cup Y|+1}, \ldots, v_{n}\right\}$.

We now show that $H \in \mathcal{H}_{n, p}^{\varepsilon, \gamma}$. As (17) implies $\Delta(H) \leqslant(1-99 \varepsilon) n p, H$ satisfies (2) for all $i \in[98 \varepsilon n]$. Note that for each $i \in[100 \varepsilon n] \backslash[98 \varepsilon n],(17)$ implies that $d_{H}\left(v_{n-i-\gamma n}\right)=0 \leqslant$ $(i-\varepsilon n) p$. Thus $H$ satisfies (3) for all $i \in[100 \varepsilon n] \backslash[98 \varepsilon n]$. Finally, for $i \in[n / 2-1] \backslash[100 \varepsilon n]$, (17) implies that $d_{H}\left(v_{i}\right) \leqslant 101 \varepsilon n p \leqslant(n-i) p-\varepsilon n p$, where the final inequality holds with room to spare. Thus $H$ satisfies $(2)$ for all $i \in[n / 2-1] \backslash[100 \varepsilon n]$. Hence, $H \in \mathcal{H}_{n, p}^{\varepsilon, \gamma}$. Therefore, $G_{n, p}$ a.a.s. contains a subgraph $H \in \mathcal{H}_{n, p}^{\varepsilon, \gamma}$ such that $G_{n, p} \backslash H$ does not contain an optimal matching.

\section{Pósa's theorem for Hamilton cycles in random graphs}

Our approach for the proof of Theorem 2 builds on the ideas of Lee and Sudakov [25], with some modifications and additional steps to account for the increased flexibility in the choice of the graph $H$ that we remove. Thus we only describe the necessary tools as well as the main steps. The corresponding proofs that we omit here can be found in the appendix. For $H \in \mathcal{H}_{n, p}^{\varepsilon}$, we rely heavily on the fact that graphs of the form $G_{n, p} \backslash H$ have good expansion properties; namely, they satisfy Proposition 12.

Whenever we consider a path $P$ on a vertex set $W$ we mean that $V(P) \subseteq W$. Let $G$ be a graph and let $P=v_{1} \ldots v_{\ell}$ be a path on $V(G)$. Let $v:=v_{1}$ and $u:=v_{\ell}$ be the endpoints of $P$. Suppose $v_{i} \in N_{G}(v)$ for some $i \neq \ell$. Then, we can also consider the path $P^{\prime}=v_{i-1} v_{i-2} \ldots v v_{i} v_{i+1} \ldots u$ in $G \cup P$. We refer to the path $P^{\prime}$ as a rotation of $P$ within $G$ with fixed endpoint $u$ and pivot $v_{i}$. We call $v_{i-1} v_{i}$ the broken edge of the rotation.

Starting from $P$, we will consider successive rotations of $P$ to obtain new paths, always leaving one of the endpoints of $P$ fixed. We only consider rotations whose broken edges are edges in the original path $P$.

For any vertex $x \in V(P)$, let $x_{P, u}^{-}$and $x_{P, u}^{+}$denote the predecessor and successor of $x$ along $P$, respectively (where $P$ is oriented towards the fixed endpoint $u$ ). Similarly, given any set $X \subseteq V(P)$, we denote $X_{P, u}^{+}:=\left\{x_{P, u}^{+}: x \in X\right\}$ and $X_{P, u}^{-}:=\left\{x_{P, u}^{-}: x \in X\right\}$. 
Let $\mathcal{R}_{G, P, u} \subseteq V(P)$ be the set of all vertices $x \in V(P)$ such that there exists a path $P_{x}$ in $G \cup P$ with endpoints $u$ and $x$ which can be obtained by taking successive rotations of $P$ within $G$ with fixed endpoint $u$. (As mentioned before, we only consider rotations whose broken edges are in $P$.) Whenever we consider a vertex $x \in \mathcal{R}_{G, P, u}$, the notation $P_{x}$ will be used to denote a path with endpoints $x$ and $u$ which can be obtained by the minimum number of rotations of $P$ (whenever there is more than one choice for $P_{x}$, we fix such a choice arbitrarily among all the possibilities). Let $R_{G, P, u}^{0}:=\{v\}$ and $R_{G, P, u}^{t}$ be the set of vertices $x \in \mathcal{R}_{G, P, u}$ such that $P_{x}$ is obtained by at most $t$ rotations.

Given any set $A \subseteq \mathcal{R}_{G, P, u}$, we denote by $R_{G, P, u}(A)$ the union of $A$ and the set of endpoints of all paths which are obtained via a single rotation of $P_{a}$ with $u$ as a fixed endpoint, for any $a \in A$.

The following observation is well-known. We include the short proof in the appendix.

Lemma 17. Let $G$ be a graph. Let $P^{\prime}$ be a path on $V(G)$ and let $P=v_{1} \ldots v_{\ell}$ be a longest path in $G \cup P^{\prime}$. Then, for all $t \geqslant 0$ we have

$$
\left|R_{G, P, v_{\ell}}^{t+1}\right| \geqslant \frac{1}{2}\left(\left|N_{G}\left(R_{G, P, v_{\ell}}^{t}\right)\right|-3\left|R_{G, P, v_{\ell}}^{t}\right|\right) .
$$

Next, we restrict ourselves to the random graph $G_{n, p}$. Given a 'large' set $A$ of endpoints obtainable via a 'small' number of successive rotations of a longest path $P$, we prove a lower bound on the number of endpoints obtainable from $A$ via one further rotation.

Lemma 18. Let $0<1 / C \ll \eta \ll \varepsilon<1$. For $p \geqslant C \log n / n$, the random graph $G=G_{n, p}$ a.a.s. satisfies the following. Let $G^{\prime}$ be a subgraph of $G$ and $P^{\prime}$ be a path on $V$. Let $P=v_{1} \ldots v_{\ell}$ be a longest path in $G^{\prime} \cup P^{\prime}$. Then, for all $A \subseteq R_{G^{\prime}, P, v_{\ell}}^{\eta \log n}$ with $|A| \geqslant \varepsilon n / 100$, we have that $\left|R_{G^{\prime}, P, v_{\ell}}(A)\right| \geqslant p^{-1} \delta_{G^{\prime}}(A)-\varepsilon n / 10$.

The proof of Lemma 18 is similar to (part of) the proof of Lemma 3.2 in [25]. For completeness, we include the details in the appendix.

We now combine the two previous results to give a lower bound on the number of endpoints which can be generated via successive rotations of a path $P$ with one fixed endpoint.

Lemma 19. Let $0<1 / C \ll \varepsilon<1$. For $p \geqslant C \log n / n$, the random graph $G=G_{n, p}$ a.a.s. satisfies the following. Let $H \in \mathcal{H}_{n, p}^{\varepsilon}(G)$ and $G^{\prime}:=G \backslash H$. Let $P^{\prime}$ be a path on $V$. For any longest path $P=v_{1} \ldots v_{\ell}$ in $G^{\prime} \cup P^{\prime}$, there exists $U \subseteq V$ with $|U| \geqslant(1 / 2+\varepsilon / 4) n$ such that, for every $v \in U$, there exists a longest path $Q_{v}$ in $G^{\prime} \cup P^{\prime}$ with endpoints $v_{\ell}$ and $v$, where $V\left(Q_{v}\right)=V(P)$.

Proof. Let $u:=v_{\ell}$. Throughout this proof we write $R^{t}$ for $R_{G^{\prime}, P, u}^{t}$ and $R(A):=R_{G^{\prime}, P, u}(A)$ for any $A \subseteq \mathcal{R}_{G^{\prime}, P, u}$. Let $\eta$ be a number such that $1 / C \ll \eta \ll \varepsilon$. Condition on the event that the following holds for all $v \in V$ :

$$
d_{G}(v)=(1 \pm \eta) n p
$$


We also condition on the event that the assertions of Proposition 12 and Lemma 18 hold for $G$. By Lemmas 10 and 18 and Proposition 12, each of these events holds a.a.s.

Note that (18) and the fact that $H \in \mathcal{H}_{n, p}^{\varepsilon}(G)$ imply that, for any set $X \subseteq V$ with $|X| \geqslant \varepsilon n / 10$

there exists a set $X^{\prime} \subseteq X$ with $\left|X^{\prime}\right| \geqslant \varepsilon n / 20$ and $\delta_{G^{\prime}}\left(X^{\prime}\right) \geqslant \min \{|X|, n / 2\} p+$ $\varepsilon n p / 2$.

Note that, since $P$ is a longest path in $G^{\prime} \cup P^{\prime}$, we have that $N_{G^{\prime}}(x) \subseteq V(P)$ for all $x \in \mathcal{R}_{G^{\prime}, P, u}$. We will consider successive rotations of $P$, keeping $u$ fixed, to derive a lower bound on the number of distinct endpoints of different longest paths in $G^{\prime} \cup P^{\prime}$ with an endpoint $u$.

By Lemma 17 together with the assertion of Proposition 12(i), for each $t \geqslant 0$, we have

$$
\left|R^{t+1}\right| \geqslant \frac{1}{2}\left(\min \left\{\frac{1}{2} \varepsilon\left|R^{t}\right| n p, \frac{1}{2} \varepsilon n(\log n)^{-1 / 4}\right\}-3\left|R^{t}\right|\right) .
$$

As $R^{0}=\left\{v_{1}\right\}$ and $\varepsilon n p / 2>\log n$, the above inequality implies that there exists $s \in \mathbb{N}$ with $s \leqslant \frac{1}{2} \eta \log n$ such that

$$
\left|R^{s}\right| \geqslant \frac{\varepsilon n}{5(\log n)^{1 / 4}}
$$

Again, by applying Lemma 17 together with the assertion of Proposition 12(ii), we obtain that $\left|R^{s+1}\right| \geqslant \varepsilon n / 10$.

Now, in order to show that $\left|R^{s+5 \varepsilon^{-1}+1}\right| \geqslant(1 / 2+\varepsilon / 4) n$, we will iteratively construct sets $Y_{0}, \ldots, Y_{5 \varepsilon^{-1}}$ as follows.

Let $Y_{0}:=R^{s+1}$. Suppose that for some $0 \leqslant j<5 \varepsilon^{-1}$ we have already constructed $Y_{j}$ with $\left|Y_{j}\right| \geqslant(j+1) \varepsilon n / 10$. We use (19) to obtain a subset $Y_{j}^{\prime} \subseteq Y_{j}$ with $\left|Y_{j}^{\prime}\right| \geqslant \varepsilon n / 20$ and $\delta_{G^{\prime}}\left(Y_{j}^{\prime}\right) \geqslant(j+1) \varepsilon n p / 10+\varepsilon n p / 2$. Let $Y_{j+1}:=R\left(Y_{j}^{\prime}\right)$. By Lemma 18, we have

$$
\left|Y_{j+1}\right|=\left|R\left(Y_{j}^{\prime}\right)\right| \geqslant p^{-1} \delta_{G^{\prime}}\left(Y_{j}^{\prime}\right)-\varepsilon n / 10 \geqslant(j+1) \varepsilon n / 10+\varepsilon n / 2-\varepsilon n / 10 \geqslant(j+2) \varepsilon n / 10 .
$$

Note that we can apply Lemma 18 as $s+1+j \leqslant s+5 \varepsilon^{-1} \leqslant \frac{1}{2} \eta \log n+5 \varepsilon^{-1} \leqslant \eta \log n$. By repeating this for $0 \leqslant j<5 \varepsilon^{-1}$, we have $\left|Y_{5 \varepsilon^{-1}}\right| \geqslant(1 / 2+\varepsilon / 4) n$.

By the construction, $Y_{5 \varepsilon^{-1}} \subseteq R^{s+5 \varepsilon^{-1}+1} \subseteq R^{\eta \log n}$. Letting $U:=R^{\eta \log n}$ concludes the proof.

Definition 20. Let $\delta>0$. We say that a connected $n$-vertex graph $G$ has property $R E(\delta)$ if one of the following holds for every path $P$ on $V(G)$ :

(i) there exists a path longer than $P$ in the graph $G \cup P$,

(ii) there exists $S_{P} \subseteq V(G)$ with $\left|S_{P}\right| \geqslant \delta n$ and a collection $\left\{T_{v}: v \in S_{P}\right\}$ of subsets of $V(G)$ with $\left|T_{v}\right| \geqslant \delta n$ for all $v \in S_{P}$ satisfying the following: for all $v \in S_{P}$ and $w \in T_{v}$, the graph $G \cup P$ contains a path $Q$ between $v$ and $w$ with $V(Q)=V(P)$. 
Lemma 21. For every $0<\varepsilon<1$ there exists $C>0$ such that, for $p \geqslant C \log n / n$, the random graph $G=G_{n, p}$ a.a.s. satisfies the following. Let $H \in \mathcal{H}_{n, p}^{\varepsilon}(G)$ and $G^{\prime}:=G \backslash H$. Then, $G^{\prime}$ satisfies $R E(1 / 2+\varepsilon / 4)$.

Proof. Recall that $G$ a.a.s. satisfies the assertions of Proposition 12 and Lemma 19. We prove that $G^{\prime}$ satisfies $R E(1 / 2+\varepsilon / 4)$ conditioned on this.

By Proposition 12(iii), $G^{\prime}$ is connected. Let $P$ be any path on $V$. We may assume that $G^{\prime} \cup P$ does not contain a path which is longer than $P$. Let one of the endpoints of $P$ be $u$. By Lemma 19, there exists $S_{P} \subseteq V$ with $\left|S_{P}\right| \geqslant(1 / 2+\varepsilon / 4) n$ and such that, for every $v \in S_{P}$, there exists a path $Q_{v} \subseteq G^{\prime} \cup P$ with endpoints $u$ and $v$ such that $V\left(Q_{v}\right)=V(P)$. For each path $Q_{v}$ we can fix $v$ and apply Lemma 19 again to obtain a set $T_{v} \subseteq V$ such that $\left|T_{v}\right| \geqslant(1 / 2+\varepsilon / 4) n$ and for every $x \in T_{v}$ there is a path $Q_{x v} \subseteq G^{\prime} \cup P$ from $x$ to $v$ with $V\left(Q_{x v}\right)=V(P)$. The result follows.

Definition 22. Let $\delta>0$ and let $G_{1}$ be a graph on $n$ vertices with property $R E(\delta)$. We say that a graph $G_{2}$ with $V\left(G_{2}\right)=V\left(G_{1}\right)$ complements $G_{1}$ if, for every path $P$ on $V\left(G_{1}\right)$, one of the following holds:

(i) there exists a path longer than $P$ in $G_{1} \cup P$,

(ii) there exist sets $S_{P}$ and $T_{v}$ as in Definition 20 and vertices $v \in S_{P}$ and $w \in T_{v}$ such that $v w$ is an edge of $G_{1} \cup G_{2}$.

Proposition 23 ([25]). Let $\delta>0$. For every $G_{1} \in R E(\delta)$ and $G_{2}$ complementing $G_{1}$, the union $G_{1} \cup G_{2}$ is Hamiltonian.

Finally, we state two lemmas which are used to complete the proof of Theorem 2. The first says that, given $G=G_{n, p}$ and $H \in \mathcal{H}_{n, p}^{\varepsilon}(G)$, the graph $G \backslash H$ complements every 'small' subgraph of $G$ which has property $R E(1 / 2+\varepsilon / 4)$. The final lemma then says that $G^{\prime}$ actually contains some such 'small' graph as a subgraph. We include the proof of Lemma 24 in the appendix.

Lemma 24. For every $0<\varepsilon<1$, there exist $C, \delta>0$ such that for $p \geqslant C \log n / n$ we have that $G=G_{n, p}$ a.a.s. satisfies the following property: for any $H \in \mathcal{H}_{n, p}^{\varepsilon}(G)$, the graph $G \backslash H$ complements all graphs $R \subseteq G$ which satisfy $R E(1 / 2+\varepsilon / 4)$ and have at most $\delta n^{2} p$ edges.

Lemma 25. For all $0<\varepsilon, \delta \leqslant 1$, there exists $C>0$ such that, for $p \geqslant C \log n / n$, the graph $G=G_{n, p}$ a.a.s. satisfies the following property. Let $H \in \mathcal{H}_{n, p}^{2 \varepsilon}(G)$. Then, $G \backslash H$ contains a subgraph with at most $\delta n^{2} p$ edges satisfying $R E(1 / 2+\varepsilon / 4)$.

Proof. Let $1 / n \ll 1 / C \ll \varepsilon, \delta$ and $1 / c<1$. Let $p^{\prime}:=\delta p$. We say that a graph $F$ on $V$ is good if it has at most $n^{2} p^{\prime}=\delta n^{2} p$ edges and, for all $H \in \mathcal{H}_{n, p^{\prime}}^{\varepsilon}$, the graph $F \backslash H$ satisfies $R E(1 / 2+\varepsilon / 4)$. Otherwise, we call it bad. Given any graph $F$ on $V$, let $\hat{F}$ be the graph obtained from $F$ by taking every edge of $F$ independently with probability $\delta$. 
Let $\hat{\mathbb{P}}$ be the measure associated with the experiment $\hat{F}$. Let $\mathbb{P}_{\text {total }}$ be the product measure obtained from considering the experiments yielding $G_{n, p}$ and $\hat{G}_{n, p}$ (i.e. with respective measures $\mathbb{P}$ and $\hat{\mathbb{P}}$ ). Note that, by definition, the edge distribution of $\hat{G}_{n, p}$ is identical to that of $G_{n, p^{\prime}}$. It follows by Lemmas 9 and 21 that $\mathbb{P}_{\text {total }}\left[\hat{G}_{n, p}\right.$ is good $]=$ $\mathbb{P}\left[G_{n, p^{\prime}}\right.$ is good $]=1-o(1)$.

Let $\mathcal{F}$ be the collection of all graphs $F$ on $V$ for which $\hat{\mathbb{P}}[\hat{F}$ is good $] \geqslant 3 / 4$. Since

$$
o(1)=\mathbb{P}_{\text {total }}\left[\hat{G}_{n, p} \text { is bad }\right] \geqslant \mathbb{P}\left[G_{n, p} \notin \mathcal{F}\right] \mathbb{P}_{\text {total }}\left[\hat{G}_{n, p} \text { is bad } \mid G_{n, p} \notin \mathcal{F}\right] \geqslant \mathbb{P}\left[G_{n, p} \notin \mathcal{F}\right] / 4 \text {, }
$$

we know that $\mathbb{P}\left[G_{n, p} \notin \mathcal{F}\right]=o(1)$ or, in other words, $\mathbb{P}\left[G_{n, p} \in \mathcal{F}\right]=1-o(1)$. Thus, from now on, we consider $G=G_{n, p}$ and condition on the event that $G \in \mathcal{F}$.

Let $H \in \mathcal{H}_{n, p}^{2 \varepsilon}(G)$. Using Lemma 8 and taking a union bound over all vertices in $V$, we have that $\hat{\mathbb{P}}\left[\hat{G} \cap H \in \mathcal{H}_{n, p^{\prime}}^{\varepsilon}\right]=1-o(1)$. Since $\hat{G}$ is good with probability at least $3 / 4$, and $\hat{G} \cap H \in \mathcal{H}_{n, p^{\prime}}^{\varepsilon}$ with probability $1-o(1)$, there exists a choice of $\hat{G}$ which satisfies these two properties. For such $\hat{G}$, by the definition of good, the graph $\hat{G} \backslash H$ satisfies $R E(1 / 2+\varepsilon / 4)$. Moreover, $\hat{G}$ has at most $\delta n^{2} p$ edges and, hence, so does $\hat{G} \backslash H$. Since $\hat{G} \backslash H \subseteq G \backslash H$, the result follows.

The proof of Theorem 2 now follows from the previous results.

Proof of Theorem 2. Let $1 / n \ll 1 / C \ll \delta \ll \varepsilon$. Condition on the assertions of Lemmas 24 and 25 holding with $\varepsilon / 2$ instead of $\varepsilon$, which happens a.a.s. We will show that for any $H \in \mathcal{H}_{n, p}^{\varepsilon}(G)$, the graph $G \backslash H$ is Hamiltonian.

Let $H$ be a graph as above. By Lemma 25, there exists a subgraph $G^{*}$ of $G \backslash H$ which has at most $\delta n^{2} p$ edges and satisfies property $R E(1 / 2+\varepsilon / 8)$. By Lemma 24 we have that $G \backslash H$ complements $G^{*}$. Therefore, Proposition 23 implies that $G \backslash H$ is Hamiltonian.

\section{Acknowledgements}

We are grateful to António Girão for some helpful discussions which led us to simplify one of our proofs.

\section{References}

[1] M. Ajtai, J. Komlós and E. Szemerédi, First occurrence of Hamilton cycles in random graphs, Cycles in graphs (Burnaby, B.C., 1982), vol. 115 of North-Holland Math. Stud., 173-178, North-Holland, Amsterdam (1985).

[2] P. Allen, J. Böttcher, J. Ehrenmüller and A. Taraz, The Bandwidth Theorem in sparse graphs, arXiv:1612.00661.

[3] J. Balogh, B. Csaba and W. Samotij, Local resilience of almost spanning trees in random graphs, Random Structures Algorithms 38 (2011), 121-139.

[4] J. Balogh, C. Lee and W. Samotij, Corrádi and Hajnal's theorem for sparse random graphs, Combin. Probab. Comput. 21 (2012), 23-55. 
[5] S. Ben-Shimon, M. Krivelevich and B. Sudakov, Local resilience and Hamiltonicity maker-breaker games in random regular graphs, Combin. Probab. Comput. 20 (2011), 173-211.

[6] S. Ben-Shimon, M. Krivelevich and B. Sudakov, On the resilience of Hamiltonicity and optimal packing of Hamilton cycles in random graphs, SIAM J. Discrete Math. 25 (2011), 1176-1193.

[7] B. Bollobás, The evolution of sparse graphs, Graph theory and combinatorics (Cambridge, 1983), 35-57, Academic Press, London (1984).

[8] B. Bollobás and A. Thomason, Random graphs of small order, Random graphs '83 (Poznań, 1983), vol. 118 of North-Holland Math. Stud., 47-97, North-Holland, Amsterdam (1985).

[9] V. Chvátal, On Hamilton's ideals, J. Combin. Theory Ser. B 12 (1972), 163-168.

[10] P. Condon, A. Espuny Díaz, A. Girão, D. Kühn and D. Osthus, Dirac's theorem for random regular graphs, arXiv:1903.05052.

[11] G. A. Dirac, Some theorems on abstract graphs, Proc. Lond. Math. Soc. 2 (1952), 69-81.

[12] P. Erdős and A. Rényi, On random matrices, Magyar Tud. Akad. Mat. Kutató Int. Közl 8 (1964), 455-461.

[13] P. Erdős and A. Rényi, On the existence of a factor of degree one of a connected random graph, Acta Math. Acad. Sci. Hungar. 17 (1966), 359-368.

[14] A. Ferber, R. Nenadov, A. Noever, U. Peter and N. Škorić, Robust Hamiltonicity of random directed graphs, J. Combin. Theory Ser. B 126 (2017), 1-23.

[15] A. Frieze and M. Krivelevich, On two Hamilton cycle problems in random graphs, Israel J. Math. 166 (2008), 221-234.

[16] D. Hefetz, A. Steger and B. Sudakov, Random directed graphs are robustly Hamiltonian, Random Structures Algorithms 49 (2016), 345-362.

[17] H. Huang, C. Lee and B. Sudakov, Bandwidth theorem for random graphs, J. Combin. Theory Ser. B 102 (2012), 14-37.

[18] S. Janson, T. Łuczak and A. Ruciński, Random graphs, Wiley-Interscience Series in Discrete Mathematics and Optimization, Wiley-Interscience, New York (2000).

[19] R. M. Karp, Reducibility among combinatorial problems, 85-103, Springer US (1972).

[20] F. Knox and A. Treglown, Embedding spanning bipartite graphs of small bandwidth, Combin. Probab. Comput. 22 (2013), 71-96.

[21] J. Komlós and E. Szemerédi, Limit distribution for the existence of Hamiltonian cycles in a random graph, Discrete Math. 43 (1983), 55-63.

[22] A. D. Koršunov, Solution of a problem of P. Erdős and A. Rényi on Hamiltonian cycles in undirected graphs, Dokl. Akad. Nauk SSSR 228 (1976), 529-532.

[23] M. Krivelevich, C. Lee and B. Sudakov, Resilient pancyclicity of random and pseudorandom graphs, SIAM J. Discrete Math. 24 (2010), 1-16. 
[24] D. Kühn, D. Osthus and A. Treglown, Hamiltonian degree sequences in digraphs, J. Combin. Theory Ser. B 100 (2010), 367-380.

[25] C. Lee and B. Sudakov, Dirac's theorem for random graphs, Random Structures Algorithms 41 (2012), 293-305.

[26] R. Montgomery, Hamiltonicity in random directed graphs is born resilient, arXiv: 1901.09605.

[27] R. Montgomery, Hamiltonicity in random graphs is born resilient, Random Structures Algorithms (to appear).

[28] R. Nenadov, A. Steger and M. Trujić, Resilience of perfect matchings and Hamiltonicity in random graph processes, Random Structures Algorithms 54 (2019), 797-819.

[29] L. Pósa, A theorem concerning Hamiltonian lines, Magyar Tud. Akad. Mat. Kutató Int. Közl. 7 (1962), 225-226.

[30] L. Pósa, Hamiltonian circuits in random graphs, Discrete Math. 14 (1976), 359-364.

[31] N. Škorić, A. Steger and M. Trujić, Local resilience of an almost spanning $k$-cycle in random graphs, Random Structures Algorithms 53 (2018), 728-751.

[32] K. Staden and A. Treglown, On degree sequences forcing the square of a Hamilton cycle, SIAM J. Discrete Math. 31 (2017), 383-437.

[33] B. Sudakov and V. H. Vu, Local resilience of graphs, Random Structures Algorithms 33 (2008), 409-433.

[34] A. Treglown, A degree sequence Hajnal-Szemerédi theorem, J. Combin. Theory Ser. B 118 (2016), 13-43.

\section{A Proofs of Lemmas 17, 18 and 24}

Proof of Lemma 17. Throughout the proof we write $R^{t}:=R_{G, P, v_{\ell}}^{t}$ and, for all $x \in V(P)$, $x^{+}:=x_{P, v_{\ell}}^{+}$and $x^{-}:=x_{P, v_{\ell}}^{-}$. Since $P$ is a longest path, we must have that $N_{G}(x) \subseteq V(P)$ for all $x \in \mathcal{R}_{G, P, v_{\ell}}$. Let $T:=\left\{x \in N_{G}\left(R^{t}\right) \backslash R^{t} \mid x^{-}, x^{+} \notin R^{t}\right\}$. It follows that if $x \in T$, then the segment of $P$ formed by $x^{-}, x$ and $x^{+}$is preserved under any sequence of $t$ rotations of $P$. Since $x \in N_{G}\left(R^{t}\right) \backslash R^{t}$, it follows that one of $x^{-}, x^{+}$must be in $R_{G, P, v_{\ell}}\left(R^{t}\right)=R^{t+1}$. Now let $T_{+}:=\left\{x^{+} \mid x \in T, x^{+} \in R^{t+1}\right\}$ and $T_{-}:=\left\{x^{-} \mid x \in T, x^{-} \in R^{t+1}\right\}$. We have that either $\left|T_{+}\right| \geqslant|T| / 2$ or $\left|T_{-}\right| \geqslant|T| / 2$. It follows that

$$
\left|R^{t+1}\right| \geqslant \frac{1}{2}|T| \geqslant \frac{1}{2}\left|N_{G}\left(R^{t}\right) \backslash R^{t}\right|-\left|R^{t}\right| .
$$

Proof of Lemma 18. Let $0<1 / C \ll \eta \ll 1 / c \ll \varepsilon<1$. We condition on the event that the following holds for all $X, Y \subseteq V$ :

$$
e_{G}^{\prime}(X, Y)=|X||Y| p \pm c \sqrt{|X||Y| n p} .
$$

Indeed, Lemma 9 implies this event a.a.s. occurs. 
Let $H:=G \backslash G^{\prime}$. We partition $P$ into $k:=\eta^{1 / 2} \log n$ vertex-disjoint intervals $P_{1}, \ldots, P_{k}$ with $V(P)=\bigcup_{i \in[k]} V\left(P_{i}\right)$, whose lengths are as equal as possible. By abusing notation, we will also view $P_{i}$ and $P$ as vertex sets. Consider any $A \subseteq R_{G^{\prime}, P, v_{\ell}}^{\eta \log n}$ with $|A| \geqslant \varepsilon n / 100$. Throughout this proof, we write $R(A):=R_{G, P, v_{\ell}}(A)$. For each $i \in[k]$, let $\hat{X}_{i} \subseteq A$ be the collection of all those vertices $x \in A$ for which some edge in $P_{i}$ is broken in the sequence of rotations resulting in $P_{x}$. Let $X_{i,+}$ and $X_{i,-}$ be the collections of all those vertices $x \in A$ such that $P_{i}$ is unbroken (i.e. it contains no broken edges) in the sequence of rotations resulting in $P_{x}$, and where $P_{x}$ (when directed from $x$ to $v_{\ell}$ ) traverses $P_{i}$ in the original and reverse order, respectively. Note that $A=\hat{X}_{i} \cup X_{i,+} \cup X_{i,-}$ for every $i \in[k]$. Let $I:=\left\{i \in[k]:\left|\hat{X}_{i}\right| \geqslant \eta^{1 / 4}|A|\right\}$.

We claim that

$$
|I| \leqslant \eta^{3 / 4} \log n
$$

Indeed, recall that each vertex in $A$ is obtained by at most $\eta \log n$ rotations of $P$. By considering the total sum of the number of rotations performed to obtain each different endpoint in $A$ we observe that

$$
\eta^{1 / 4}|A| \cdot|I| \leqslant|A| \cdot \eta \log n
$$

which implies (22).

Claim 26. We have $e_{H}^{\prime}(A, V) \geqslant|A||V \backslash R(A)| p-\eta^{1 / 5} n^{2} p$.

Proof. To prove this, note that, since $P$ is a longest path, we have $e_{G^{\prime}}^{\prime}(A, V \backslash P)=0$. Hence,

$$
e_{H}^{\prime}(A, V \backslash P)=e_{G}^{\prime}(A, V \backslash P) \text {. }
$$

Throughout this proof, for any $X \subseteq V(P)$ we write $X^{+}:=X_{P, v_{\ell}}^{+}$and $X^{-}:=X_{P, v_{\ell}}^{-}$. For vertices $v_{j} \in P_{i} \cap P_{i}^{-}$and $x \in X_{i,+}$, if $x v_{j+1}$ is an edge in $G^{\prime}$, then we have $v_{j} \in R(A)$. In other words, $x$ has no edges to $\left(P_{i} \cap P_{i}^{+}\right) \backslash R(A)^{+}$in the graph $G^{\prime}$. By a similar argument, a vertex $x \in X_{i,-}$ has no edges to $\left(P_{i} \cap P_{i}^{-}\right) \backslash R(A)^{-}$in $G^{\prime}$. Thus, we have

$$
e_{G^{\prime}}^{\prime}\left(X_{i,+},\left(P_{i} \cap P_{i}^{+}\right) \backslash R(A)^{+}\right)=0 \quad \text { and } \quad e_{G^{\prime}}^{\prime}\left(X_{i,-},\left(P_{i} \cap P_{i}^{-}\right) \backslash R(A)^{-}\right)=0 .
$$

As $G^{\prime}=G \backslash H$, this implies that all edges of $G$ between $X_{i, *}$ and $\left(P_{i} \cap P_{i}^{*}\right) \backslash R(A)^{*}$ belong to $H$, for $* \in\{+,-\}$. As $P_{i} \cap P_{i}^{*}$ and $P_{i}^{*}$ differ by exactly one vertex, by (23) and (24) 
we have

$$
\begin{aligned}
e_{H}^{\prime}(A, V) & \geqslant e_{G}^{\prime}(A, V \backslash P)+\sum_{* \in\{+,-\}} \sum_{i=1}^{k}\left(e_{G}^{\prime}\left(X_{i, *},\left(P_{i} \cap P_{i}^{*}\right) \backslash R(A)^{*}\right)\right. \\
& \geqslant e_{G}^{\prime}(A, V \backslash P)+\sum_{* \in\{+,-\}} \sum_{i=1}^{k}\left(e_{G}^{\prime}\left(X_{i, *},\left(P_{i} \backslash R(A)\right)^{*}\right)-4 k n\right. \\
& \stackrel{(21)}{\geqslant}|A||V \backslash P| p-c \sqrt{|A| n^{2} p}+\sum_{* \in\{+,-\}} \sum_{i=1}^{k}\left(\left|X_{i, *}\right|\left|P_{i} \backslash R(A)\right| p-c \sqrt{\left|X_{i, *}\right|\left|P_{i}\right| n p}\right) \\
& \geqslant|A||V \backslash P| p+\sum_{* \in\{+,-\}} \sum_{i=1}^{k}\left|X_{i, *}\right|\left|P_{i} \backslash R(A)\right| p-4 c \sqrt{k n^{3} p} \\
& \geqslant|A||V \backslash P| p+\sum_{i=1}^{k}\left|A \backslash \hat{X}_{i}\right|\left|P_{i} \backslash R(A)\right| p-4 c \sqrt{k n^{3} p},
\end{aligned}
$$

where we used that $\left|P_{i}\right| \leqslant|P| / k+1$ in the penultimate inequality, and the fact that $A=\hat{X}_{i} \cup X_{i,+} \cup X_{i,-}$ in the final inequality. By the definition of $I$, we have $\left|A \backslash \hat{X}_{i}\right| \geqslant$ $\left(1-\eta^{1 / 4}\right)|A|$ for all $i \in[k] \backslash I$. Therefore, we have

$$
\begin{aligned}
e_{H}^{\prime}(A, V) & \geqslant|A||V \backslash P| p+\left(1-\eta^{1 / 4}\right)|A| p \sum_{i \in[k] \backslash I}\left|P_{i} \backslash R(A)\right|-4 c \sqrt{k n^{3} p} \\
& \stackrel{(22)}{\geqslant}|A||V \backslash R(A)| p-2 \eta^{1 / 4}|A| n p-4 c \sqrt{k n^{3} p} \\
& \geqslant|A||V \backslash R(A)| p-\eta^{1 / 5} n^{2} p .
\end{aligned}
$$

We obtain the final inequality as $p \geqslant \log n / n$ implies $\sqrt{k n^{3} p} \leqslant \eta^{1 / 4} n^{2} p$. This proves the claim.

On the other hand, we have $e_{G^{\prime}}^{\prime}(A, V) \geqslant|A| \delta_{G^{\prime}}(A)$ and, by (21), we have

$$
e_{G}^{\prime}(A, V) \leqslant|A| n p+c \sqrt{n^{3} p} \leqslant(1+\eta)|A| n p .
$$

Therefore,

$$
e_{H}^{\prime}(A, V)=e_{G}^{\prime}(A, V)-e_{G^{\prime}}^{\prime}(A, V) \leqslant(1+\eta)|A| n p-|A| \delta_{G^{\prime}}(A) .
$$

Combining this with Claim 26 gives the desired inequality,

$$
|R(A)| \geqslant \delta_{G^{\prime}}(A) p^{-1}-\eta n-\frac{\eta^{1 / 5} n^{2}}{|A|} \geqslant \delta_{G^{\prime}}(A) p^{-1}-\varepsilon n / 10
$$


Proof of Lemma 24. Let $1 / C \ll \delta \ll \varepsilon$. Let $\mathcal{G}$ be the family of all subgraphs of the form $G \backslash H$, for all $H \in \mathcal{H}_{n, p}^{\varepsilon}$. (Note that we have $H \in \mathcal{H}_{n, p}^{\varepsilon}$ here instead of $H \in \mathcal{H}_{n, p}^{\varepsilon}(G)$, because this is more convenient for the argument below. But this results in the same family $\mathcal{G}$.)

The probability that the assertion of the lemma fails is

$$
\begin{aligned}
p^{*} & :=\mathbb{P}\left[\bigcup_{R \in R E(1 / 2+\varepsilon / 4), e(R) \leqslant \delta n^{2} p}\left(\{R \subseteq G\} \cap\left\{\text { some } G^{\prime} \in \mathcal{G} \text { does not complement } R\right\}\right)\right] \\
& \leqslant \sum_{R \in R E(1 / 2+\varepsilon / 4), e(R) \leqslant \delta n^{2} p} \mathbb{P}\left[\text { some } G^{\prime} \in \mathcal{G} \text { does not complement } R \mid R \subseteq G\right] \mathbb{P}[R \subseteq G],
\end{aligned}
$$

where the union and sum are taken over all labelled graphs $R$ on $V$ which have property $R E(1 / 2+\varepsilon / 4)$ and at most $\delta n^{2} p$ edges.

Let $R$ be a fixed graph on $V$ with property $R E(1 / 2+\varepsilon / 4)$ and at most $\delta n^{2} p$ edges. Let $P$ be a fixed path on $V$. If in $R \cup P$ there is a path longer than $P$, then condition (i) of Definition 22 is already satisfied, so we can assume that there is no such path in $R \cup P$. Then, by the definition of property $R E(1 / 2+\varepsilon / 4)$, we can find a set $S_{P} \subseteq V$ and, for every $v \in S_{P}$, a corresponding set $T_{v, P} \subseteq V$, as in Definition 20. We can assume that for each $v \in S_{P}$ we have that $e_{R}\left(v, T_{v, P}\right)=0$, as otherwise $R$ complements itself and there is nothing more to prove. For each $S \subseteq S_{P}$ with $|S|=\varepsilon n / 8$ let $\mathcal{H}_{S} \subseteq \mathcal{H}_{n, p}^{\varepsilon}$ be the collection of graphs $H \in \mathcal{H}_{n, p}^{\varepsilon}$ for which every $v \in S$ is of the form $v_{i}$ for $i \geqslant n / 2$ with respect to the ordering of $V(H)$ given in Definition 1. Note that $\bigcup_{S \subseteq S_{P}:|S|=\varepsilon n / 8} \mathcal{H}_{S}=\mathcal{H}_{n, p}^{\varepsilon}$. Thus, given any such $S \subseteq S_{P}$ and $H \in \mathcal{H}_{S}$, we have $d_{H}(v) \leqslant(1 / 2-\varepsilon) n p$ for all $v \in S$. For each such $S \subseteq S_{P}$ and all $v \in S$, define $T_{v, P, S}:=T_{v, P} \backslash S$. Note that $\left|T_{v, P, S}\right| \geqslant(1 / 2+\varepsilon / 8) n$.

Fix $S \subseteq S_{P}$ and $v \in S$. Since $\left|T_{v, P, S}\right| \geqslant(1 / 2+\varepsilon / 8) n$, by Lemma 8 we have

$$
\mathbb{P}\left[e_{G}\left(v, T_{v, P, S}\right) \leqslant n p / 2 \mid R \subseteq G\right] \leqslant e^{-\Omega_{\varepsilon}(n p)} .
$$

Since $S$ is disjoint from all sets of the form $T_{v, P, S}$, these events are independent for different vertices. Thus, using that $|S|=\varepsilon n / 8$, we can see that

$$
\mathbb{P}\left[e_{G}\left(v, T_{v, P, S}\right) \leqslant n p / 2 \text { for all } v \in S \mid R \subseteq G\right] \leqslant e^{-\Omega_{\varepsilon}\left(n^{2} p\right)} .
$$

Note that if there exists $v \in S$ such that $e_{G}\left(v, T_{v, P, S}\right)>n p / 2$, then for each $H \in \mathcal{H}_{S}$ we have $e_{G \backslash H}\left(v, T_{v, P, S}\right)>n p / 2-(1 / 2-\varepsilon) n p>0$. Therefore, if some $G^{\prime} \in \mathcal{G}$ does not complement $R$, there must exist some path $P$ on $V$ and some $S \subseteq S_{P}$ with $|S|=\varepsilon n / 8$ such that all of the vertices of $S$ have fewer than $n p / 2$ neighbours in $T_{v, P, S}=T_{v, P} \backslash S$. Note that there are at most $n \cdot n$ ! choices for the path $P$ and $2^{n}$ choices for the set $S$. Taking the union bound over all choices of the path $P$ and the set $S$, by (26) we have

$\mathbb{P}\left[\right.$ some $G^{\prime} \in \mathcal{G}$ does not complement $\left.R \mid R \subseteq G\right] \leqslant n 2^{n} n ! e^{-\Omega_{\varepsilon}\left(n^{2} p\right)}=e^{-\Omega_{\varepsilon}\left(n^{2} p\right)}$. 
Combining this with (25), we have

$$
\begin{aligned}
& p^{*} \leqslant e^{-\Omega_{\varepsilon}\left(n^{2} p\right)} \sum_{R \in R E(1 / 2+\varepsilon / 4),} e_{e(R) \leqslant \delta n^{2} p} \mathbb{P}(R \subseteq G) \\
& \leqslant e^{-\Omega_{\varepsilon}\left(n^{2} p\right)} \sum_{k=1}^{\delta n^{2} p}\left(\begin{array}{c}
\left(\begin{array}{c}
n \\
2
\end{array}\right) \\
k
\end{array}\right) p^{k} \leqslant e^{-\Omega_{\varepsilon}\left(n^{2} p\right)} \sum_{k=1}^{\delta n^{2} p}\left(\frac{e n^{2} p}{k}\right)^{k} \\
& \leqslant e^{-\Omega_{\varepsilon}\left(n^{2} p\right)}\left(\delta n^{2} p\right)\left(\frac{e}{\delta}\right)^{\delta n^{2} p} \leqslant e^{-\Omega_{\varepsilon}\left(n^{2} p\right)} e^{O\left(\delta n^{2} p \log (1 / \delta)\right)} \\
& =o(1),
\end{aligned}
$$

where the penultimate inequality holds since $\left(e n^{2} p / k\right)^{k}$ is monotone increasing in the range $1 \leqslant k \leqslant \delta n^{2} p$. 